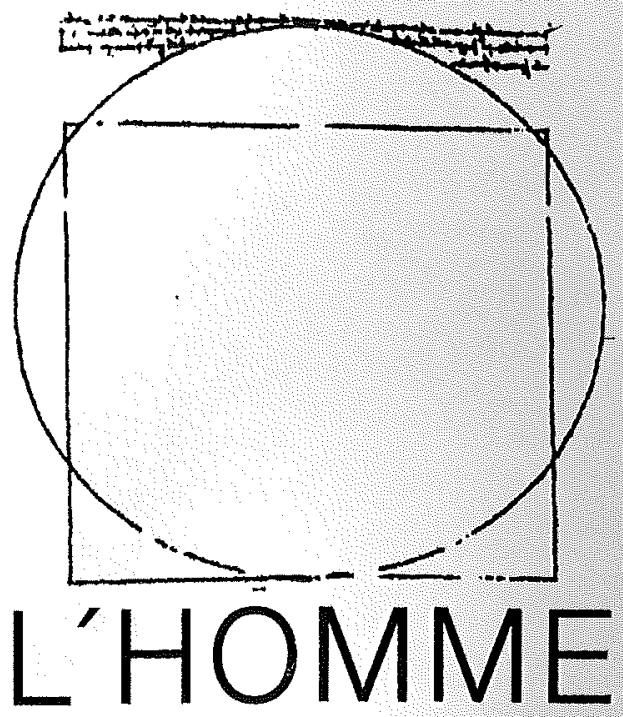

EUROPEAN REVIEW OF FEMI NIST HISTORY REVUE EUROPÉ ENNE D'HISTOIRE FÉMINISTE EUROPÄISCHE ZEITSCHRIFT FÜR FEMINISTISCHE GESCHICH TSWISSENSCHAFT EUROPEAN REVIEW OF FEMINIST HISTORY REVUE EUROPÉENNE D'HISTO IRE FÉMINISTE EUROPÄISCH E ZEITSCHRIFT FÜR FEMINISTI

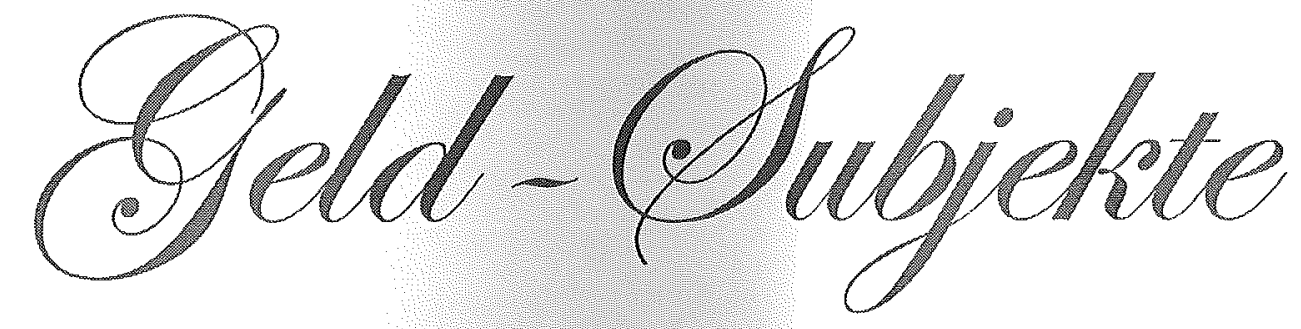

Sonderdruck

im Buchhandel nicht erhältlich

22. Jg. Heft 2 


\section{Schuld und Schulden. Zürich, 1842}

Mischa Suter

Der „Rechtstrieb“, wie in einigen Regionen der Schweiz die Zwangsexekution bei Geldforderungen genannt wurde, verband verschiedene juristische, moralische und symbolische Referenzsysteme. Dies zeigt ein ungewöhnlicher, auf den 1. Juli 1842 datierter Brief. Geschrieben wurde er von Felix Escher, einem Maler in Zürich, an Hans Heinrich Schinz, einen ehemaligen Schuldenschreiber. Escher wirft Schinz vor, er habe ein Vollstreckungsverfahren zu rasch vollzogen und ihn damit in Schwierigkeiten gebracht:

Der Rechtstrieb besteht nicht darin, den Schuldner zu verdächtigen, \& zu verleumden, ihn ganz \& gar auszusaugen, besonders wenn man überzeugt ist, daß er sein Möglichstes thut, werfen Sie einen Blick auf den Naturtrieb, würde Gott gegen Thnen \& der übrigen Menschheit in gleichem Maaße (sic) handeln wie Sie gegen mich, so müßte der Naturtrieb \& mit ihm die ganze gute Mutter Natur erstarren, die ganze Welt müßte das Schicksal Sodoms erfahren. Armuth sündet nicht, nur Laster, daß ich von solchem frey bin, das weiß ich und mein gutes Gewißen ...

Die biblische Sündenstadt Sodom wird bemüht, um den Rechtstrieb gegen den von Gott eingerichteten „Naturtrieb“ und das gute Gewissen des Schuldners gegen die üble Nachrede, das die Zwangsvollstreckung bewirke, auszuspielen. Escher fährt fort:

I Staatsarchiv Zürich (STAZH) X 347.6, Sendschreiben des Felix Escher an Hans Heinrich Schin 1. Juli 1842; Orthografie im Original. Für entscheidende Hinweise danke ich Dr. Barbara Stadler vom Staatsarchiv Zürich, für ihr präzises Gegenlesen Sandra Maß, Kirsten Bönker und Michaela Hafner, für die hilfreiche Kritik Brigitta Bernet und Anna Joss. 
Sie sind nur Altschuldenschreiber, es ist aber noch Einer der auch Ihre Schulden aufschreibt, welcher älter ist als Sie, es ist der Ewige zu dem ich bethen kann \& bethen darf „Vergib mir meine Schulden \& bewahre mich vor dem Laster". Ich bin fest überzeugt daß er mir meine Schulden vergeben wird \& sollte es auch dauren bis zum grossen Rechtsstillstand [im] Jenseits; obgleich die Lasterhaften, Sträflinge \& Sodomiten bisweilen ihre Netze \& Pfeile gegen mich auswerfen um mich zu fangen ...

Der Verfasser setzt dem Vollstreckungsverfahren religiöse und sexuelle Normen entgegen; er nennt sich verfolgt und in seiner sexuellen Integrität bedroht. Der Schuldenschreiber beaufsichtige die Zahlung monetärer Schulden, aber seine moralische Schuld wiederum würde von Gott registriert. Der Brief evoziert das Vaterunser und erwartet im Jenseits den „Rechtsstillstand“: ein juristischer Begriff für die Zeiträume rund um Ernten und Feiertage, in denen ein Vollstreckungsverfahren routinemäßig unterbrochen wurde.

Das Schreiben bezieht die christliche Norm der Vergebung auf monetäre Schulden und rückt die Durchsetzung rechtlicher Verbindlichkeit in die Nähe einer Vergewaltigung. Im Brief wird die Behauptung, dass seine heterosexuelle Männlichkeit durch Homosexuelle bedrängt werde, noch weiter gesteigert: Der "Sodomiter" sei „dem Teufel verschrieben“, ihn erwarte „der ewige Concurs!". Zuletzt beschimpft der Verfasser den Altschuldenschreiber Schinz als „Sodomiten“. Das zweiseitige Schreiben ist mit Zeichnungen versehen: ein Engel, ein Kreuz, ein Teufel auf der Vorderseite, auf der Rückseite zwei Männer beim Analverkehr.

Der Text spricht von der Ungerechtigkeit einer hartherzig vollzogenen Zwangsvollstreckung, um dann den Schuldenschreiber als moralisch verkommene Person anzugreifen. Hauptsächlich ging es dabei um letzteres; das Schreiben verfolgte den Zweck, jemanden als homosexuell zu diffamieren, wie die anschließenden Gerichtsprozesse zeigen, die sich von einer Ehrverletzungsklage in einen Kriminalfall wegen „widernatürlicher Wollust" wendeten. Der Verfasser hatte ähnliche Briefe an verschiedene Personen gesandt. Auch das vorliegende Aktenstück war nicht an Schinz selbst, sondern an dessen Ehefrau geschickt worden. ${ }^{2}$ Zudem malte Escher Mauerschriften und bot per Zeitungsinserat weitere Pamphlete zum Verkauf an. ${ }^{3}$ Schinz verklagte Escher wegen Verleumdung. Doch während des Prozesses änderte sich die Situation: Das Gericht fand im Lauf der Untersuchung Anlass, den einstigen Schuldenschreiber der „widernatürlichen Wollust" zu verdächtigen. Der Fall wurde an das Kriminalgericht Zürich

2 Vgl. STAZH YY 25.23, Protokoll Kriminalgericht Zürich, 30. März 1843, 578.

3 Vgl. Tagblatt der Stadt Zürich Nr. 193, 12. Juli 1842, 986; Nr. 194, 13. Juli 1842, 990; Nr. 196, 15. Juli 1842, 1002; Nr. 197, 16. Juli 1842, 1006. In Nr. 196, 15. Juli 1842, 1002, erschien zudem ein von Schinz selbst aufgegebenes Inserat, das bekannt gab, er habe in Bezug auf jenes Sendschreiben Anklage erhoben. Zu den Mauerschriften vgl. STAZH YY 25.23, Protokoll Kriminalgericht Zürich, 30. März 1843, 579 . weitergewiesen, das Schinz für dieses Vergehen verurteilte, und die Berufungsinstanz bekräftigte abermals das Urteil. ${ }^{4}$ Dass Escher mit einem Schmähbrief gegen Schinz vorgegangen war, könnte mit einer tatsächlich vollzogenen Zwangsvollstreckung zu tun haben, durch die er sich ungerecht behandelt gefühlt hätte. Das Schreiben deutet in diese Richtung, und im Prozess betonte die Verteidigung, es habe ein „förmlicher Complott statt gefunden", 5 denn eine "große Mehrzahl von Personen, denen seine Berufsthätigkeit ein Schreck ist", hätte „ein großes Interesse“, Schinz „von Zürich wegzusprengen". 6 Allerdings arbeitete Schinz zum Zeitpunkt des Verfahrens seit rund zehn Jahren nicht mehr als Schuldenschreiber, sondern als Geschäftsagent. ${ }^{7}$ Zudem blieb in den Verhandlungsprotokollen die Motivlage unklar und die Strategie der Verteidigung erfolglos. Das Gericht hatte Schinz’ Vergehen der „Sodomie“ im Blick, nicht den Anlass der Denunziation.

Hier soll auch nicht dieser Anlass weiterverfolgt, sondern hinter die Intention des Briefschreibers zurückgetreten werden, um aufzuzeigen, welche Verknüpfungen jener Text anstellt. Der Verfasser verwendete ein Vokabular - und darauf liegt hier das Augenmerk -, das monetäre Schulden mit moralischer Schuld verband. Der Brief rief eine bestimmte Rhetorik für eine höhnische Parodie auf, und so lässt sich nach dem argumentativen Register dieser Parodie fragen. Gegen die offene Geldschuld wurde die sexuelle Grenzüberschreitung gesetzt. Indem die Hetzschrift in das Narrativ einer rigiden Zwangsvollstreckung gekleidet war, behauptete sic ein konfligierendes Verhältnis zwischen moralischer Verpflichtung und monetärer Zahlungsverbindlichkeit, dem in abgeschwächter Form weit verbreitete ambivalente Haltungen und Befürchtungen entsprachen. Gesellschaftliche Vorstellungen maskuliner Souveränität waren darin zentral. Im Schuldverhältnis wurde der Subjektstatus von SchuldnerInnen angetastet und transformiert. Das Vollstreckungsverfahren regulierte die sozialen Beziehungen zwischen GläubigerInnen und DebitorInnen, es wirkte auf diese Beziehungen ein, wurde von den Beteiligten als Mittel eingesetzt und rief dabei widersprüchliche Effekte hervor

\section{Selbstführung durch Verfahren}

Wenn die Schmähschrift polemisch christliche Werte mit Geldschulden in Beziehung setzte, so hatte vier Jahrzehnte zuvor ein Theologe die pazifizierende Dimension eines

\footnotetext{
4 Das Obergericht vergrößerte das Strafmaß von sechs Monaten auf ein Jahr Gefängnis und dreijährigen Kantonsverweis. Für die Urteile der ersten und zweiten Instanz vgl. STAZH YY 25.23, Protokoll gen Kantonsverweis. Für die Urteile der ersten und zweiten Instanz vgl. STAZH YY 25.23, Protokoll
Kriminalgericht Zürich, 30. März 1843, 604; STAZH YY 10.38, Kriminalprotokoll Obergericht ZïKriminalgericht Zürich, 30. Mä
rich 1843, 16. Mai $1843,957$.

5 STAZH YY 10.38, Kriminalprotokoll Obergericht Zürich 1843, 16. Mai 1843, 940

6 STAZH YY 25.23, Protokoll Kriminalgericht Zürich, 30. März 1843, 579.

7 Als ,alt Schuldenschreiber" wird Schinz erstmals erwähnt in: Hans Jakob Holzhalb, Verzeichniß der Stadt-Bürgerschaft von Zürich auf das Jahr 1834, Zürich 1834, 173 .
} 
Schuldennachlasses betont. Die Formel „Vergieb uns unsre Schulden, wie wir unsern Schuldnern vergeben“, schrieb 1800 der Archidiakon des Zürcher Grossmünsters, Johannes Tobler, sei eine "Thatenmaxime“, die „allen Revolutionen vorböge" und eine „[g]utartige Hierarchie“" bewahren würde. Die Gläubiger sollten jenen, die „Geld, Dienstleistungen, Frohnen, Zinse, Zehenden schuldig sind“, je nach Umständen, „[a]ber immer so, daß einiche Güte darunter waltet, - bald Wartensfrist bewilligen bald die Schuld heruntersezen (sic), - bald sie gänzlich durchstreichen und schenken. ... Und damit hätte man Herzen gewinnen, - gleichsam kaufen können - o gewiß nicht sehr theuer und doch von unsaglichem Werth. " Indem er vorschlug, Herzen "gleichsam kaufen" zu lassen, zeigte sich der Theologe Tobler durchaus sensibilisiert für die merkantile Unterseite paternalistischer Verpflichtung. Hier wurde für eine situative Stundung der Schulden plädiert. Ein solcher fallweiser Ausgleich war der regelhaften Prozedur der Zwangsvollstreckung keineswegs einfach entgegengesetzt; vielmehr bildeten Verfahrenslogik und situatives Handeln zwei komplementäre Momente.

Den Rechtstrieb, ein Regulativ zur Gewährleistung des „öffentlichen Kredits“, zeichnete in seiner formalen Grundstruktur eine bemerkenswerte Kontinuität aus. Charakteristisch war die administrative, Schlankheit' des Verfahrens. Da die betreffende Geldforderung häufig gar nicht strittig war, meldeten die GläubigerInnen schriftlich ihren Anspruch beim Schuldenschreiber, der durch einen Unterbeamten die Mahnung zustellen ließ. In einer Serie von Fristen wurde die Zahlungsaufforderung in der Kirche verlesen und schließlich Werte verpfändet oder der Konkurs eingeleitet. Zeitgenössische Juristen äußerten sich fasziniert darüber, wie selten der Gang vor Gericht angestrebt wurde. ${ }^{10}$ Das Verfahren stützte sich stark auf Selbstführung, auf ein konformes Verhalten in einem autoregulativen Operationsmodus, der kaum Foren der Auseinandersetzung beanspruchte. Bei dieser grundsätzlich stabilen Regierungstechnik nahmen im Lauf des 19. Jahrhunderts lokale Momente zu. Jene Aufwertung des Lokalen lässt sich in einen Interpretationsrahmen integrieren, der ein ,ruling at a distance als konstitutiv für liberales Regieren sieht. ${ }^{11}$ Als 1830/31 im Kanton Zürich eine liberale Regierung an die Macht kam, forderte eine Vielzahl landschaftlicher Petitionen, den Rechtstrieb dezentral zu organisieren und auf Gemeindeebene anzusiedeln. ${ }^{2}$

8 Johannes Tobler, Der schöne Vaterlandsfriede. (Gleichsam) aus Einem Prinzip hergeleitet, in: ders., Gutartige Hierarchie. Armenbesorgung, und die schöne Friedensbitte, Zürich 1800, 11-14, 12f.

Für die weit verbreitete Formel vom Vollstreckungswesen als Rahmenstruktur des „öfentlichen Kredits" vgl. z. B. Rechenschaftsbericht des Obergerichts an den Grossen Rath des Standes Zürich über das Jahr 1844, Zürich 1845, 20.

1o Vgl. Fr. von Wyß, Die Schuldbetreibung nach schweizerischen Rechten, in: Zeitschrift für schweizerisches Recht, 7 (1858), 3-114, 85f.; ders., Schuldbetreibung, in: Johann Caspar Bluntschli Hg., Deutsches Staats-Wörterbuch, Bd. 9, Stuttgart/Leipzig 1865, 258-264, 259f.

II Für eine solche Interpretation liberaler Regierungstechnik des 19. Jahrhunderts vgl. Patrick Joyce, The Rule of Freedom. Liberalism and the Modern City, London/New York 2003, insbes. 100.

I2 Die vom Grossen Rat animierten 270 Petitionen (von denen 75 den "Rechtstrieb" thematisierten) finden sich in STAZH KIII 258.3 (Nr. 201-270); KIII 258.3a (Nr. 1-75); K III 259.1
Zuvor hatte - außer bei geringen Beträgen, deren Eintreibung die Gemeinden regelten - ein einziger Schuldenschreiber mit vier Beamten von der Stadt aus die Verfahren für den ganzen Kanton abgewickelt. ${ }^{13}$ Die Petitionen machten vor allem die hohen Gebühren und Portokosten geltend, aber nach der Umgestaltung zeigte sich, dass die moderierende Funktion lokaler Kräfte ein entscheidender Grund war. Ab 1832 wurden die Schuldenschreiber auf Bezirksebene eingesetzt; weil dadurch elf Beamte die Geschäfte betrieben und sich die Gebühren teilen mussten, wurde das Amt für Hans Heinrich Schinz, den Empfänger des eingangs zitierten Schandbriefs, uninteressant. ${ }^{14}$ Unablässig war indes die Klage der Aufsichtsbehörde, dass deren Unterbeamten die Verfahren verschleppten. ${ }^{15}$ Die niederen Beamten vor Ort suchten die Kosten, welche pauperisierte SchuldnerInnen dem kommunalen Armenwesen bereiteten, zu vermeiden, beabsichtigten die Risiken von Dominoeffekten unter wechselseitig verschuldeten Haushalten zu begrenzen und zögerten vor allem bei der „Versilberung", das heißt der Veräußerung der verpfändeten Wertgegenstände. So wurde im Jahr der Wirtschaftskrise 1849 gegenüber der Aufsichtsbehörde betont, dass es ,in vielen Fällen dem Gemeindeammann bei dem besten Willen nicht möglich sei, die Versilberung nach gesetzlicher Vorschrift vorzunehmen, wenn er nicht ... dem Schuldner seinen Credit vollends entziehen wolle ...". 16 Zusätzlich zu den „Rechtsstillständen“, den erwähnten sakral und agrarwirtschaftlich bestimmten Verfahrensunterbrechungen, die nicht weniger als 16 Wochen des Jahres umfassten, ${ }^{17}$ mäßigten insgeheim elastische Fristen das Prozedere. Der Rechtstrieb mit

(Nr. 133-200); K III 259.1a (Nr. 76-132). Eine thematische Einteilung und Nummerierung be [David Ulrich], Uebersicht der der Verfassungs-Commission gemachten Eingaben, in so fern dieselben sich nicht zunächst auf die Staatsverfassung, sondern auf die verschiedenen Zweige der Verwaltung, der Justizpflege und der Gesetzgebung beziehen, Zürich 1831, 31. Ausführlich zu den Petitionen vgl. Barbara Weinmann, Eine andere Bürgergesellschaft. Klassischer Republikanismus und Kommunalismus im Kanton Zürich im späten 18. und 19. Jahrhundert, Göttingen 2002, 204-267, insbes. 238f.

13 Vgl. Gesetz betreffend die Form und Kosten des Rechtstriebs, 17. Dezember 1803, in: Officielle Sammlung der von dem Großen Rathe des Cantons Zürich gegebenen Gesetze und gemachten Verordnungen, und der von dem Kleinen Rath emanierten allgemeinen Landes- und Polizey-Verordnungen, Bd.1, Zürich 1804, 193-203, 193, $\$ 1$.

I4 Dies suggeriert ein von Schinz zusammen mit den vier Schuldenboten verfasstes Schreiben, das sich mit dem für 1832 geplanten Gesetz auseinandersetzt: STAZH P.5.2.1, 28. November 1831.

is So der Rechenschaftsbericht des Obergerichts an den Großen Rath des Standes Zürich über das Jahr 1834, Zürich 1835, 13; über das Jahr 1835, 11f; über das Jahr 1837, 13f,; über das Jahr 1838, 13f,

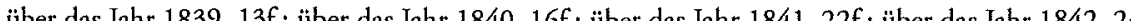
(18) 854 dort Statistiken über die ermahnten Beamten, 29-37.

I6 STAZH P 5.2,2, Obergericht des Standes Zürich, Aufsicht der Gemeindeammänner durch die Schuldenschreiber, 6. Januar 1849.

17 Vgl. Zürcherischer Schreiblkalender auf das Jahr 1842, Zürich 1841; Gottfried von Meiss, Das PfandRecht und der Pfand- oder Betreibungs-Proceß in seinem ganzen Umfang: Nach den Gesetzen und der Uebung des Eidgen. Cantons Zürich/Ein civilrechthlicher Versuch, Zürich 1821, 128 
seiner sequenziellen Logik stellte mehr als einen juristischen Rahmen. Er bildete ein Werkzeug der Machtausübung, das Leute einsetzten: ein Moment der Markierung in nerhalb und mittels liberal gestalteter Kräfteverhältnisse zwischen handelnden Subjekten. Zugespitzt formuliert: Wie die Schmähschrift, die den Empfänger verletzen sollte, waren die zugestellten Vollstreckungsurkunden Texte, die auf Wirkungen zielten - sie stellten Briefe dar, die bezweckten, die EmpfängerInnen zu treffen und unter Druck zu setzen.

\section{Widersprüchliche Subjektivierung}

Die Markierung, die eine Zwangsvollstreckung bedeutete, versetzte Personen in verschiedene Zustände, die eng juristisch definierte Dimensionen überstiegen. Die Geschlechterordnung bildete dabei einen zentralen Angelpunkt. Genau hier entstanden inkonsistente rechtliche Situationen und konfliktreiche Grenzzustände. Mit Blick auf den eingangs zitierten Schandbrief interessieren speziell die Auffassungen von Männlichkeit, die im Rechtstrieb auf dem Spiel standen. Bei Frauen geschah die Zustellung wie auch der Empfang einer Zwangsvollstreckung unter Geschlechtsvormundschaft, für niedrige Konsumschulden wurden verheiratete Frauen hingegen direkt behaftet. ${ }^{18}$ Wenn auch das von einer Frau in die Ehe eingebrachte Vermögen, das Frauengut, nominell im Konkursfall ihres Ehemannes bevorzugt behandelt wurde, so musste sie in der Lage sein, ihre Ansprüche durchzusetzen. Bei Männern bestand eine Bandbreite restringierter rechtlicher Identitäten: Den Gegenpol zum „aufrechtstehenden Schuldner" ${ }^{\text {"19, }}$ wie verschiedene staatsrechtliche Texte die Zahlungsfähigkeit umschrieben, bildete der „Fallit“, der im „Falliment" genannten Konkursverfahren abgestürzt war und die Bürgerrechte verloren hatte. Zeitgenössische Rechtsexperten bemühten sich zwar, den mit Depotenzierungsängsten verbundenen Topos vom „bürgerlichen Tod“ zu entkräften. ${ }^{20}$ Indes räumte ein Jurist ein, es solle ein „Fallit“ seinen Gläubigern „so zu sagen mit Leib

I8 Vgl. David von Wyss, Politisches Handbuch für die erwachsene Jugend der Stadt und Landschaft Zürich, Zürich 1796, 172.

19 Der Ausdruck findet sich u. a. im Konkordat der eidgenössischen Tagsatzung vom 15. Juni 1804, das die Zwangsvollstreckung von Schuldnern über Kantonsgrenzen hinweg behandelte. In der Beratung zur Bundesverfassung von 1848 wurde zunächst die Formulierung „der zahlungsfähige ... Schuldner“ verwendet, worauf die Gesandtschaft des Kantons Schwyz sich erfolgreich dafür auseprach, den fï̈verwenct wor hern vels wo Individuum unter gewissen Verhaltnissen zahlungstahig sein könne, ohne daß ihm das Prädikat de ökonomischen Integrität zugesprochen werden dürfe." Auszug aus dem Abschiede der ordentlichen eidgenössischen Tagsatzung des Jahres 1847, IV. Theil: Verhandlungen, betreffend die Revision des Bundesvertrages, [Bern ca, 1848], 271.

20 Vgl. Brigitta Bernet, „Der bürgerliche Tod“: Entmündigungsangst, Psychiatriekritik und die Krise de liberalen Subjektentwurfs um 1900, in: dies. u. a., Zwang zur Ordnung. Psychiatrie im Kanton Zürich, 1870-1970, Zürich 2007, 117-153. und Gut verfallen seyn, bis sie ihre völlige Befriedigung gefunden haben" ${ }^{21}$ Maskuline Souveränität definierte sich dabei über eheliche Herrschaft: Falliten verloren juristisch die Kontrolle über ihre Ehefrauen, die unter Amtsvormundschaft gestellt wurden. Ein Gesetz von 1810 stellte fest, „daß die Eheweiber von Falliten, nach dem Falliment ihrer Männer, meist ohne Aufsicht bleiben, und oft ... mit ihrem geretteten Weibergut nach Belieben schalten und walten, wordurch (sic) für den öffentlichen Credit sowohl, als für die bürgerliche Ordnung sehr nachtheilige Folgen entstehen ...". ${ }^{22}$ Dabei kollidierte die obrigkeitliche Vormundschaft mit den Ansprüchen von Ehemännern: „Himmelschreiende Ungerechtigkeiten werden oft den Eheweibern und Kindern der Falliten durch die Bevogtigungen beigebracht", fand eine Druckschrift von 1840, während der Familienvater dazu „nichts sagen darf", weil er „die Ansprüche an Gott, Himmel und Seligkeit und an die Gemeindrechte verwirkt und verloren" hätte. ${ }^{23}$

Gewissermaßen spiegelbildlich führt die Unglücksrhetorik solcher Texte die Mündigkeit als eine männliche Subjektivierungsweise vor - der Eingriff in die Souveränität durch eine Zwangsvollstreckung bedeutete einen Angriff auf den liberalen Subjektentwurf. ${ }^{24}$ Dieser Entwurf folgte einem weit verbreiteten und häufig kommentierten bürgerlichen Narrativ, das wirtschaftliches und persönliches Scheitern verknüpfte und als - manchmal realen - Endpunkt nur mehr Flucht oder Selbstmord übrig ließ. ${ }^{25}$ So suchte ein Schuldner „seine bürgerliche Ehre als das edelste Kleinod zu bewahren“, wie sich eine Petition von 1830 ausdrückte. ${ }^{26}$ Und doch blieb dieses Narrativ weder unidirektional noch unwidersprochen. Verschuldung und Zwangsvollstreckung bildeten weitere, zum Teil konträre und variantenreiche Subjektivierungen aus. Widersprüchliche rechtliche Identitäten trugen zu dieser Varianz bei. Die hier diskutierten Beispiele legen nahe, dass Verfahrenslogiken immer wieder temperiert, durchkreuzt und vertragliche Ansprüche mit symbolischen Verbindlichkeiten gekontert wurden. SchuldnerInnen rechtfertigten sich, indem sie argumentativ weitere normative Horizonte hinzuzogen oder gegenläufige Referenzsysteme in Anschlag brachten; in beiden Fällen wurde die Bedeutung grundlegend polysemischer Begriffe wie ,Schuld' unter der Hand verschoben. Die Unglücksthetorik spielte wiederholt die Homonymie von Schuld und

2I Friedrich Ludwig Keller, Ueber den Rechtszustand der Falliten, in: Monatschronik der zürcherischen Rechtspflege, 1 (1833), 113-121, 118.

22 Gesetz, betreffend die Bevogtigung der Eheweiber von Falliten, 13. Dezember 1810, in: Officielle Sammlung der von dem Großen Rathe des Cantons Zürich gegebenen Gesetze und gemachten Verordnungen, und der von dem Kleinen Rath emanierten allgemeinen Landes- und Polizey-Verordnungen, Bd. 4, Zürich 1811, 383 .

23 STAZH P.5.2.1, Heinrich Jäggli, Dachslern, und Konrad Leimbacher, Neftenbach, an den Grossen Rat von Zürich, 27./30. Januar 1840

24 Vgl. Bernet, Tod, wie Anm, 20

25 Diese beiden verbreiteten Topoi nennt eine Bittschrift mit Bezug auf die Inhaftierung von Schuldnern in: STAZH P.5.2.2, Jakob Bänz, Wülflingen, an den Grossen Rat von Zürich, o. D. (ca. Dezember 1849).

26 Vgl. STAZH K III 258.3a, Nr. 40, Wetzikon, \$30, Dezember 1830. 
Verschuldung wechselseitig aus: Wer unverschuldet in Schulden geraten sei, den treffe keine Schuld und er sei deshalb von den Schulden freizusprechen. „Was muß aus einer solchen Gegend werden", schrieb der Verfasser einer Eingabe, der gegen die verkürzten Fristen des Rechtstriebsgesetzes von 1832 opponierte, „wenn sie eine so grosse Zahl unverschuldeter Weise verarmter, güterloser und nichts mehr zu verlieren habender Männer unter sich hat?" ${ }^{37}$ Die Eingabe mit leicht drohendem Unterton hob in diesem Fall Recht und Moral als diskordante Register hervor. Eine andere, grelle Verknüpfung von Recht und Moral stellte der eingangs zitierte Hetzbrief gegen den Altschuldenschreiber Schinz dar. In der Schmähschrift wurde der als missbräuchlich deklarierte Rechtstrieb gegen den „Naturtrieb" gesetzt und mit der „widernatürlichen Wollust“ parallelisiert. Die behauptete Unnatürlichkeit dieses speziellen Vollstreckungsfalls kontrastierte umgekehrt mit der sequenziellen, naturalisierten Systemlogik des auf Selbstführung abstützenden Verfahrens der Zwangsvollstreckung. Solche Register standen offen für verschiedene, widersprüchliche und im vorliegenden Fall höhnisch-parodistische Gebrauchsformen.

27 STAZH P.5.2.1, Rudolf Spiess u. a., Vorsteherschaft von Uhwiesen an den Grossen Rat, 4. Februar 1834 (Hervorhebung im Original). 